Research Article

\title{
Antibacterial Activity of Sodium Hypochlorite Gel versus Different Types of Root Canal Medicaments Using Agar Diffusion Test: An In Vitro Comparative Study
}

\author{
Mohamed El Sayed (D), ${ }^{1,2}$ Nikta Ghanerad $\left(\mathbb{D},{ }^{1}\right.$ Fatemeh Rahimi $\left(\mathbb{D},{ }^{1}\right.$ Mahin Shabanpoor $(\mathbb{D})$, \\ and Zeinab Shabanpour iD ${ }^{1}$ \\ ${ }^{1}$ Ajman University, College of Dentistry, Restorative Dentistry Department, P.O. Box 346, Ajman, UAE \\ ${ }^{2}$ Mansoura University, Faculty of Dentistry, Endodontic Department, El-Gomhoureya Street, El-Mansoura city, \\ 35516 El-Dakahleya, Egypt
}

Correspondence should be addressed to Mohamed El Sayed; elsayednada@yahoo.com

Received 5 March 2020; Revised 28 August 2020; Accepted 29 October 2020; Published 28 November 2020

Academic Editor: Maurizio D’Amario

Copyright (c) 2020 Mohamed El Sayed et al. This is an open access article distributed under the Creative Commons Attribution License, which permits unrestricted use, distribution, and reproduction in any medium, provided the original work is properly cited.

\begin{abstract}
Aim. This study aimed to evaluate the antibacterial effect of sodium hypochlorite gel and four types of intracanal medicaments. Materials and Methods. The agar diffusion method was used to evaluate the antibacterial effect of five medicaments (sodium hypochlorite gel $(\mathrm{NaOCl})$, chlorhexidine gel $(\mathrm{CHX})$, calcium hydroxide paste $(\mathrm{CH})$, Ledermix, and Diapex plus) against Enterococcus faecalis (E. faecalis), Staphylococcus aureus (S. aureus), and Escherichia coli (E. coli). The zone of inhibition around each medicament was measured in millimeters, after 48 hours of incubation at $37^{\circ} \mathrm{C}$. The antibacterial effects of medicaments against each microbial strain and the sensitivity of microorganisms towards each medicament were compared using the one-way ANOVA and Games-Howell post hoc tests. The level of significance was set to $p<0.05$. Results. All medicaments showed variable inhibition zones for all bacterial strains except Diapex Plus which showed no antibacterial activity. NaOCl gel exhibited the most significant inhibition zones for all bacterial strains followed by CHX gel, Ledermix, and $\mathrm{CH}$. However, the effect of $\mathrm{CHX}$ and $\mathrm{CH}$ paste against $S$. aureus was statistically similar, while the effect of $\mathrm{CH}$ against $E$. faecalis was significantly higher than the Ledermix. Conclusion. Sodium hypochlorite gel displayed the highest antibacterial activity among tested medicaments and can be recommended as a potent intracanal medicament. Chlorhexidine gel showed a significantly higher antibacterial effect when compared with Ledermix and calcium hydroxide. Calcium hydroxide demonstrated stronger antibacterial activity against E. faecalis than Ledermix. Diapex Plus exhibited no antibacterial effect.
\end{abstract}

\section{Introduction}

Bacteria and their toxic byproducts are the main causes of pulpal and periapical diseases [1]. The most important goals of successful endodontic treatment are to eliminate the microorganisms confined in the root canal system and prevent the regrowth of residual microorganisms [2]. Infected root canals have a complex microbial flora consisting of cocci, rods, spirochetes, filaments, and sometimes fungi that are typically found together in endodontic flare-ups and cases with posttreatment apical periodontitis $[3,4]$. Facultative anaerobic bacteria such as Enterococcus faecalis (E. faecalis) and Staphylococcus aureus (S. aureus) have been thought to be the most resistant species in the oral cavity that may cause root canal treatment failure $[5,6]$. E. faecalis can survive in root canals as a single organism without the support of the other bacteria [7]. S. aureus can remain viable in dentinal tubules for prolonged periods because of their resistance to drying and temperature changes [8]. Escherichia coli (E. coli) is an anaerobic Gram-negative bacillus that 
is commonly associated with persistent periapical infections [9], and its endotoxins play an important role in the induction and perpetuation of periradicular inflammatory lesions of experimental cats [10].

Different instrumentation techniques and irrigation protocols have been recommended for cleaning and disinfecting the root canal system, especially in cases with severe endodontic infections [11]. However, due to the structural complexity of root canals and the limitations of irrigation solutions, it is difficult to fully remove microorganisms from the root canal system [12, 13]. Furthermore, the deep penetration of microorganisms into dentinal tubules makes their elimination difficult $[14,15]$. Therefore, the use of an intracanal medicament with broad-spectrum antibacterial activity was necessary to complete disinfection of the prepared root canal system, especially for cases with persistence apical periodontitis $[14,15]$.

Diverse endodontic medicaments are existing with a variable degree of antimicrobial activity, such as nonsetting calcium hydroxide $\left[\mathrm{Ca}(\mathrm{OH})_{2}\right]$ and chlorhexidine gluconate (CHX). Ledermix is currently a popular root canal medicament consisting of $1 \%$ triamcinolone acetonide (corticosteroid) and 3.2\% demeclocycline-calcium (tetracycline antibiotic) in a polyethylene glycol base. It has an anti-inflammatory function to alleviate pain associated with symptomatic apical periodontitis and prevent acute exacerbation of chronic apical periodontitis [15, 16]. Diapex Plus (DiaDent, Seoul, Korea) is an alternative intracanal medicament recently launched on the market. It is a premixed paste made up of iodoform $(40.4 \%), \mathrm{Ca}$ $(\mathrm{OH})_{2}(30.2 \%)$, and hydrophobic silicone oil (22.4\%). According to the manufacturer, this product has a strong antibacterial effect and can be used to disinfect the root canals and to treat periapical lesions of endodontic origin. Sodium hypochlorite $(\mathrm{NaOCl})$ gel is available on the market as a Clorox bleaching pen gel (Clorox, USA) for cleaning cloths. The composition of this bleaching material as follows: boehmite (3-7\%), sodium hypochlorite $(0.5-2 \%)$, sodium silicate $(0.5-1.5 \%)$, and sodium petroleum sulfonate $(0.5-1.5 \%)$. However, as a trade secret, the manufacturer has withheld the exact percentage concentration of sodium hypochlorite [17]. The $\mathrm{NaOCl}$ gel can be used as intracanal medication and lubricant during the root canal instrumentation. As far as our knowledge is concerned, no previous studies have been conducted to determine the antimicrobial activity of sodium hypochlorite gel as an intracanal medicament.

Antimicrobial effectiveness of endodontic materials can be tested either in vitro or in vivo. Agar diffusion test is still the most widely used in vitro tool for evaluating the antimicrobial activity of these materials, despite its drawbacks such as lack of standardization of inoculum density, adequate culture medium, agar viscosity, plate storage conditions, size and number of specimens per plate, and time and temperature of incubation [18]. This test is simple to use and inexpensive and preserves the chemical properties of the materials tested [19]. Numerous studies on the bactericidal effect of currently available intracanal medicaments are contradictory and incomplete, and little studies have been performed on the antibacterial activity of sodium hypochlorite gel. Therefore, this study aimed to use the agar diffusion test to evaluate and compare the antibacterial activity of sodium hypochlorite gel, calcium hydroxide paste, chlorohexidine gel, Ledermix, and Diapex Plus against S. aureus, E. faecalis, and E. coli. The null hypothesis of the current study is that there are no significant differences between the antibacterial effects of experimental medicaments against all selected microorganisms.

\section{Materials and Methods}

2.1. Experimental Intracanal Medicaments. Five intracanal medicaments were tested: sodium hypochlorite gel (Clorox Bleach Pen Gel, Clorox, USA), 2\% chlorhexidine gel (Conspsis Scrub, Ultradent Products Inc., USA), nonsetting calcium hydroxide paste (Metapaste, Meta Biomed Co., Ltd, Korea), Ledermix (Riemser Pharma GMH, Germany), and DiaPex Plus (DiaDent Group, Korea). Table 1 shows the compositions of the selected intracanal medicaments and their manufacturers.

2.2. Experimental Microorganisms. The antimicrobial activity of the experimental medicaments has been tested against the following microorganisms: S. aureus, E. faecalis, and E. coli. Table 2 shows the type, source, morphotype, and manufacturer of each selected microorganism.

2.3. Inoculum Suspension Preparation. All strains have grown aerobically from frozen stock cultures in brain-heart infusion broth (BHI-Difco Laboratories, Detroit MI) at $37^{\circ} \mathrm{C}$ for a 24-hour incubation period and checked for microbial growth by turbidity changes. The turbidity of the broth culture for each microbial strain was then diluted using sterile saline until obtaining a suspension with 0.5 turbidities on the McFarland scale.

2.4. Agar Diffusion Test. Agar well diffusion tests were performed in $90 \mathrm{~mm}$ diameter Petri dishes containing Mueller Hinton Agar (BBL 211438 Becton Dickinson, Sparks, MD, USA) to a depth of $4 \mathrm{~mm}$ for all bacterial strains except $E$. faecalis, for which sheep blood agar medium was used (Merck, Darmstadt, Germany). Two agar plates were used to test the antimicrobial activity of the medicaments against one microbial strain. The top surface of each agar plate was flooded with $200 \mu \mathrm{l}$ inoculum suspension of the selected microbial strain and then dried at $37^{\circ} \mathrm{C}$ for 15 minutes. In the first agar plate, two equidistance wells of 5 $\mathrm{mm}$ diameter and $4 \mathrm{~mm}$ depth were punched using a sterile glass Pasteur pipette, while, in the second agar plate, three equidistance wells of similar diameters and depths were punched. A sterile pipette was used to place $50 \mu \mathrm{l}$ of each medication within each plate's respective well. The sodium hypochlorite gel and calcium hydroxide paste have been put in the wells of the first plate. Diapex Plus, Ledermix, and chlorohexidine gel have been put in the wells of the second plate. The plates were kept at room temperature for two hours to obtain the prediffusion of the medicament through 
TABLE 1: Experimental intracanal medicaments.

\begin{tabular}{|c|c|c|c|}
\hline Medicaments & Composition & Manufacturer & Lot. no. \\
\hline $\begin{array}{l}\text { Clorox Beach } \\
\text { Pen }\end{array}$ & $\begin{array}{l}\text { Sodium hypochlorite }(0.5-2 \%) \text {, boehmite }(3-7 \%) \text {, } \\
\text { sodium silicate }(0.5-1.5 \%) \text {, and sodium petroleum } \\
\text { sulfonate }(0.5-1.5 \%)\end{array}$ & $\begin{array}{l}\text { Clorox, Bleach Pen Gel for White, Clorox, } 1221 \\
\text { Broadway Oakland, CA 94612, USA }\end{array}$ & $137533.002 \mathrm{~J}$ \\
\hline $\begin{array}{l}\text { Consepsis } \\
\text { scrub }\end{array}$ & $2 \%$ chlorhexidine gluconate & $\begin{array}{l}\text { Ultradent Products, Inc., } 505 \text { W. } 10200 \text { S. South } \\
\text { Jordan, UT 84095, USA }\end{array}$ & B94CR \\
\hline Ledermix & $\begin{array}{c}\text { Demeclocycline calcium }(30.21 \mathrm{mg} / \mathrm{g}) \text {, triamcinolone } \\
\text { acetonide }(10 \mathrm{mg} / \mathrm{g}) \text {, macrogol } 400 \text {, macrogol } 3000 \text {, } \\
\text { zinc oxide, silicon dioxide, calcium chloride- } \\
\text { dihydrate, trolamine, sodium calcium edetate, and } \\
\text { sodium sulphate }\end{array}$ & $\begin{array}{l}\text { Riemser Pharma GMH, An der Wiek 7, } 17493 \\
\text { Greifswald-Insel Riems, Germany }\end{array}$ & 350860 \\
\hline Metapaste & $\begin{array}{c}\text { Calcium hydroxide, barium sulphate, and } \\
\text { polypropylene glycol }\end{array}$ & Meta Biomed Co., Ltd,, South Korea & MPS1708281 \\
\hline Diapex Plus & $\begin{array}{l}\text { Iodoform }(35-40 \%), \mathrm{Ca}(\mathrm{OH}) 2(20-30 \%) \text {, and } \\
\text { hydrophobic polydimethylsiloxane }(20-30 \%)\end{array}$ & $\begin{array}{l}\text { DiaDent Group International, } 16 \\
\text { Osongsaengmyeong 4-ro, Osong-eup, Heungdeok- } \\
\text { gu, Cheongju-si, Chungcheongbuk-do, Korea }\end{array}$ & WX346100 \\
\hline
\end{tabular}

TABLE 2: Bacterial strains used as indicators of antimicrobial activity, their source and morphotype.

\begin{tabular}{lccc}
\hline Microorganisms & Source & Morphotype & Manufacturer \\
\hline Staphylococcus aureus (S. aureus) & ATCC 6538 & Gram-positive, cocci, facultative anaerobes & Microbiologics, Saint Cloud, \\
Enterococcus faecalis (E. faecalis) & ATCC 29212 & Gram-positive, rod-shaped, facultative anaerobes & Minnesota, USA \\
Escherichia coli (E. coli) & ATCC 25922 & Gram-negative, rod-shaped, facultative anaerobes & \\
\hline
\end{tabular}

the agar medium and then were incubated for 48 hours under aerobic conditions at $37^{\circ} \mathrm{C}$. The entire experiment was replicated ten times for each bacterial strain, for which five all experimental medicaments were tested.

\subsection{Negative and Positive Growth Controls}

2.5.1. Negative Growth Controls (Three Agar Plates). In the experimental plates, two agar plates were prepared but without microorganisms and the experimental medicaments were placed in the prepared wells. The third plate had neither bacteria nor sealer.

2.5.2. Positive Growth Controls (Eight Agar Plates). To ensure that the microbial life cycle did not become inactive during the experimental study, each two agar plates were streaked with an individual test microorganism only without medicaments.

2.6. Measuring the Size of Inhibition Zones. Growth inhibition zones around each medicament were evidenced by the lack of bacterial colonization (clearing of agar) adjacent to each agar well. The most uniform diameter segment of the inhibition zone was measured with an endodontic millimeter ruler (Hu-Friedy Mfg. Co., Chicago, IL 60618-5935, USA), and the $6 \mathrm{~mm}$ diameter of the well was included in the measurement (Figure 1). All of the measurements above this value were considered indicative of significant inhibition of the bacterial growth. The inhibition zones with wide diameter were interpreted

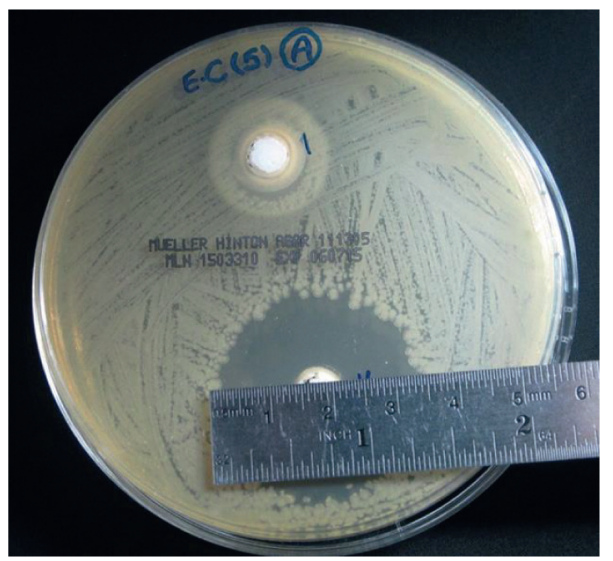

FIgURE 1: Measuring the inhibition zone using a metal ruler.

to indicate a higher antibacterial activity of the involved medicaments. At the end of the experiment, ten measurements for each inhibition zone around each medicament and against one type of microbial strain were obtained.

2.7. Statistical Analysis. Data were analyzed using version 20 of the SPSS software program (IBM, Armonk, NY, USA). The antibacterial effects of medicaments against each microbial strain and the sensitivity of microorganisms towards each medicament were compared using the One-way ANOVA and Games-Howell post hoc tests. $P$ value $<0.05$ was considered statistically significant. 

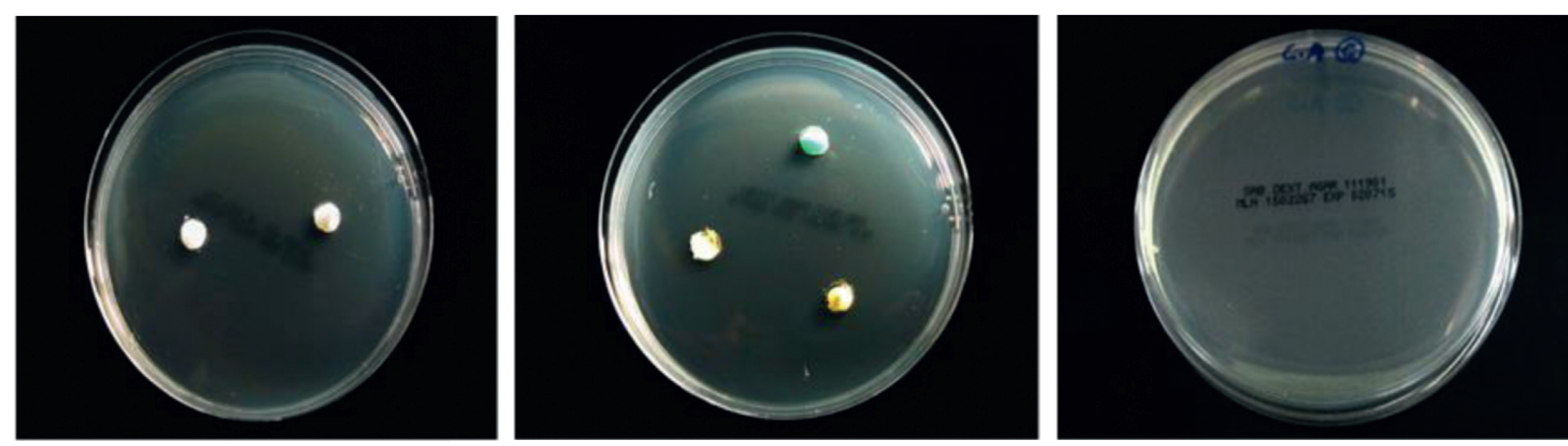

(a)
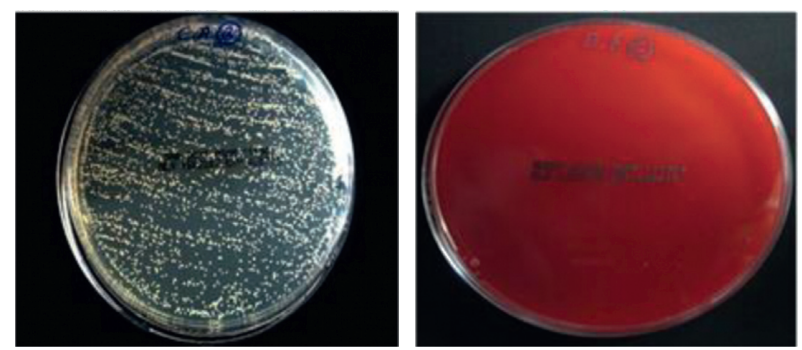

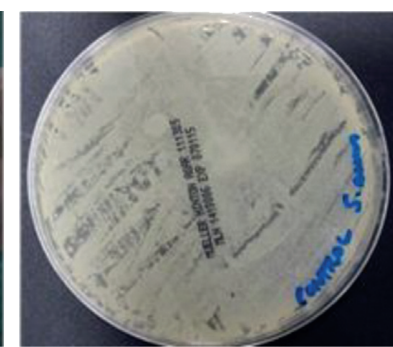

(b)

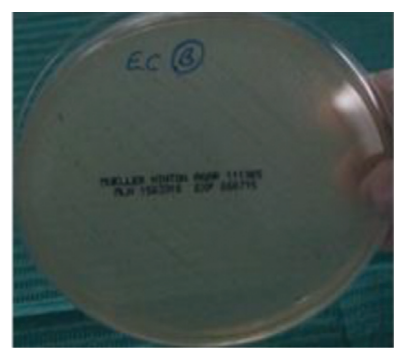

Figure 2: Agar plates for growth controls: (a) Negative growth controls; (b) Positive growth controls.

\section{Results}

There was no bacterial growth in the negative control plates, whereas the positive control plates showed noticeable and even bacterial growth (Figure 2). The mean diameters of inhibition zones developed against the tested microorganisms by the medications are shown in Table 3 and illustrated in Figure 3. The experimental medicaments showed variable diameters of inhibition zones against the tested microorganisms (Figure 4) except for the Diapex Plus that did not display any antibacterial activity against tested microorganisms.

The results of the one-way ANOVA test showed significant differences between the mean values of inhibition zones diameters produced by the tested medications against each bacterial strain $(P<0.05)$. Sodium hypochlorite gel and chlorhexidine gel had the largest inhibition zone diameters against all microorganisms. However, no significant difference was observed between the chlorohexidine gel and Ledermix regarding their antimicrobial effect against $S$. aureus. The antimicrobial activity of calcium hydroxide against $E$. faecalis was significantly higher than Ledermix and its efficacy against $E$. coli and $S$. aureus was significantly less than Ledermix $(P<0.05)$.

It was necessary to evaluate the sensitivity of microorganisms towards each medicament. There were significant differences between the tested microorganisms regarding their sensitivity towards each medicament. The highest inhibition zone around sodium hypochlorite gel occurred with E. faecalis $(34.87 \pm 1.31 \mathrm{~mm})$, followed in descending order by E. coli $(31.43 \pm 2.14 \mathrm{~mm})$ and $S$. aureus $(30.68 \pm 1.81 \mathrm{~mm})$. The highest inhibition zone with chlorohexidine gel occurred with $S$. aureus $(27.83 \pm 1.02 \mathrm{~mm})$ followed by $E$. coli $(24.40 \pm 0.82 \mathrm{~mm})$ and E. faecalis $(20.93 \pm 077 \mathrm{~mm})$.

Regarding the Ledermix, the most extensive inhibition zone occurred with $S$. aureus $(28.25 \pm 1.87 \mathrm{~mm})$, followed by E. coli $(14.23 \pm 0.53 \mathrm{~mm})$ and E. faecalis $(9.05 \pm 0.75 \mathrm{~mm})$. Nonsetting calcium hydroxide resulted in the most significant zone of inhibition with E. faecalis $(18.15 \pm 0.51 \mathrm{~mm})$, followed in descending order by $S$. aureus $(14.45 \pm 1.01 \mathrm{~mm})$ and E. coli $(12.27 \pm 1.13 \mathrm{~mm})$.

\section{Discussion}

The interest of researchers for studying antimicrobial properties of root canal irrigating solutions and medicaments has increased over the years, as they become more aware of the significance of the removal of microorganisms from the root canal system $[2,20]$. In the current study, the species of microorganisms were chosen to represent microorganisms typically isolated from untreated and previously treated infected root canal systems such as E. faecalis [5, 6], E. coli $[9,10,21]$, and S. aureus $[5,6]$.

Intracanal medicaments have a long history of use as interim appointment dressings to reduce interappointment pain, to decrease the number of microorganisms, and prevent their regrowth and to render the canal contents inert [15]. Evaluation of the antibacterial effectiveness of different intracanal medicaments is needed to understand clearly the role of these medicaments in the treatment of apical periodontitis with variable types of microorganisms.

In this research, calcium hydroxide paste was chosen, as it has antimicrobial properties and mineralization induction capability [22]. However, it has limited action against certain microorganisms, particularly E. faecalis [23] and biofilms 
TABle 3: Comparisons of mean diameters of inhibition zones $(\mathrm{mm})$ formed by the tested medicaments against each bacterial strain.

\begin{tabular}{lccccc}
\hline \multirow{2}{*}{ Medicaments } & \multicolumn{3}{c}{ Mean diameter of growth inhibition zones $( \pm \mathrm{SD})$} & ANOVA $(P$ value $)$ \\
& Plates number & S. aureus & E. faecalis & E. coli & \\
\hline Sodium hypochlorite gel & 10 & $30.68 \pm 1.81^{\mathrm{A1} 1^{*}}$ & $34.87 \pm 1.31^{\mathrm{A} 2}$ & $31.43 \pm 2.14^{\mathrm{A} 1}$ & 0.000 \\
Chlorhexidine gel & 10 & $27.83 \pm 1.02^{\mathrm{B} 1}$ & $20.93 \pm 0.77^{\mathrm{B} 2}$ & $24.40 \pm 0.82^{\mathrm{B} 3}$ & 0.000 \\
Ledermix & 10 & $28.25 \pm 1.87^{\mathrm{B} 1}$ & $9.05 \pm 0.75^{\mathrm{C} 2}$ & $14.23 \pm 0.53^{\mathrm{C} 3}$ & 0.000 \\
Calcium hydroxide & 10 & $14.45 \pm 1.01^{\mathrm{C} 1}$ & $18.15 \pm 0.51^{\mathrm{D} 2}$ & $12.27 \pm 1.13^{\mathrm{D} 3}$ & 0.000 \\
Diapex Plus & 10 & $0.00^{\mathrm{D}}$ & $0.00^{\mathrm{E}}$ & $0.00^{\mathrm{E}}$ & - \\
ANOVA $(P$ value $)$ & & 0.000 & 0.000 & 0.000 & - \\
\hline
\end{tabular}

${ }^{*}$ Games-Howell post hoc test: different uppercase letters (columns) and different uppercase numbers (rows) indicate statistically significant differences $(P<0.05)$ among the tested medicaments against the same microorganism and among antimicrobial effects of each medicament against tested microorganisms, respectively.

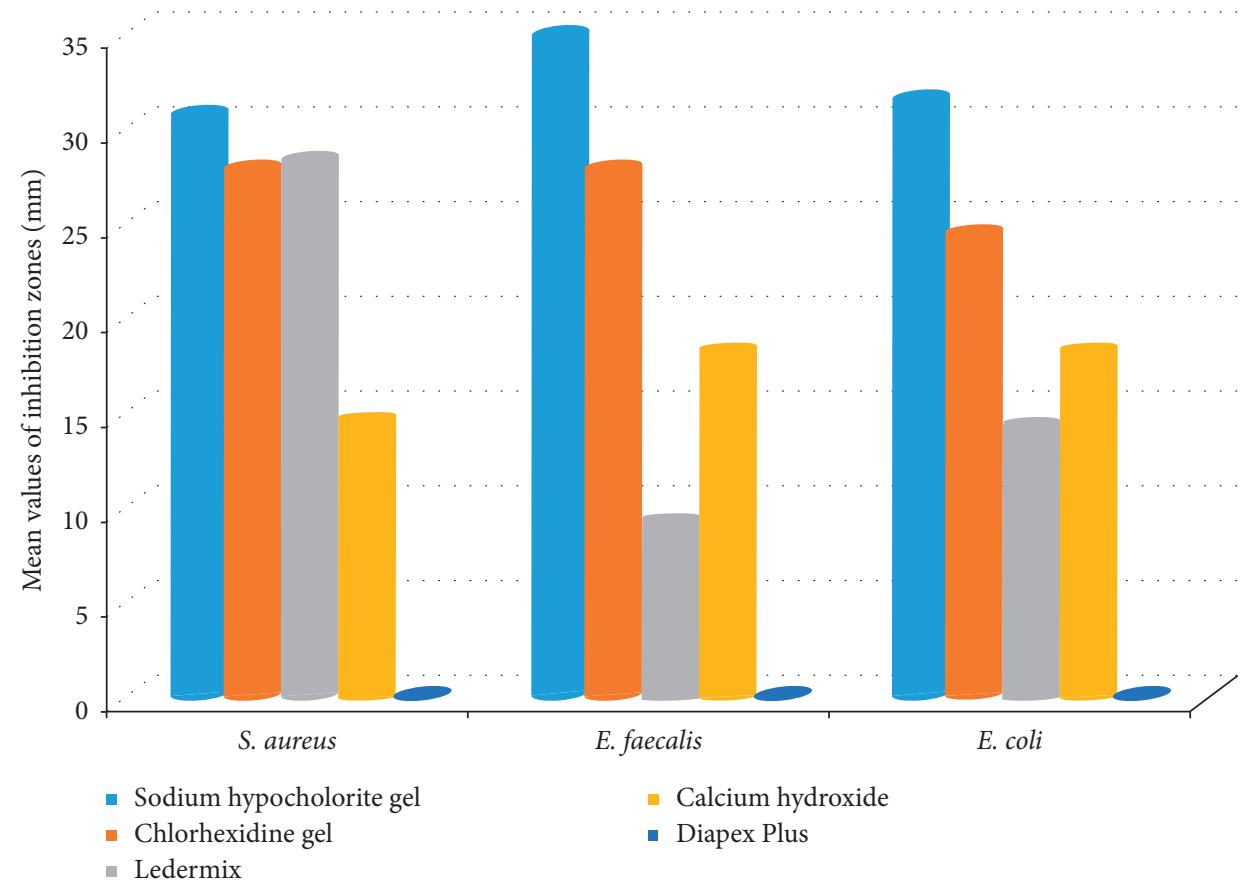

Figure 3: Mean values of the zone diameters of microbial growth inhibition according to the tested medicaments.

[24]. In addition, some researchers questioned the effectiveness of calcium hydroxide in reducing microbial numbers, even after prolonged contact with the root canal [13]. To overcome the antimicrobial limitation of calcium hydroxide, some manufacturers have added iodoform to increase its action. Diapex Plus is one of those combinations and was used in the current study as there is little knowledge about its antimicrobial activity. Chlorhexidine gel was another root canal medicament that was selected in this study as it has an excellent antimicrobial activity $[25,26]$. It can be used in the treatment of inflammatory root resorption [16] and did not affect the apical seal of the root canal $[27,28]$. Some authors suggested the use of $2 \%$ chlorhexidine gel as a root canal medication instead of calcium hydroxide [29]. However, it is not an effective intracanal barrier [30] and may reduce the success of root canal treatment [31]. Corticosteroid and antibiotic combinations have been used as an intracanal medicament due to their anti-inflammatory action [32]. Such combinations can relieve pain associated with acute apical periodontitis and prevent acute exacerbation of chronic apical periodontitis [32]. In the present research, one of those combinations (Ledermix) was used to study its antimicrobial effect against the three tested microorganisms. Ledermix paste contains an antibiotic, 3.2\% demeclocycline-calcium, and a corticosteroid, $1 \%$ triamcinolone acetonide, in a polyethylene glycol base [33]. Both triamcinolone and demeclocycline can diffuse through dentinal tubules and cementum to reach the periapical tissues [15]. Various studies have confirmed the effectiveness of Ledermix as an intracanal medicament [24, 34, 35].

It is currently important to look for an intracanal drug with good antimicrobial activity against variable species of endodontic microorganisms. $\mathrm{NaOCl}$ is known to be the best irrigating solution that has had the most active antimicrobial activity against the most possible root canal microorganisms [36]. This solution, however, has high toxicity to the tissue when it is apically extruded. The use of $\mathrm{NaOCl}$ gel can reduce the risk of apical extrusion and the overall side effects of its 

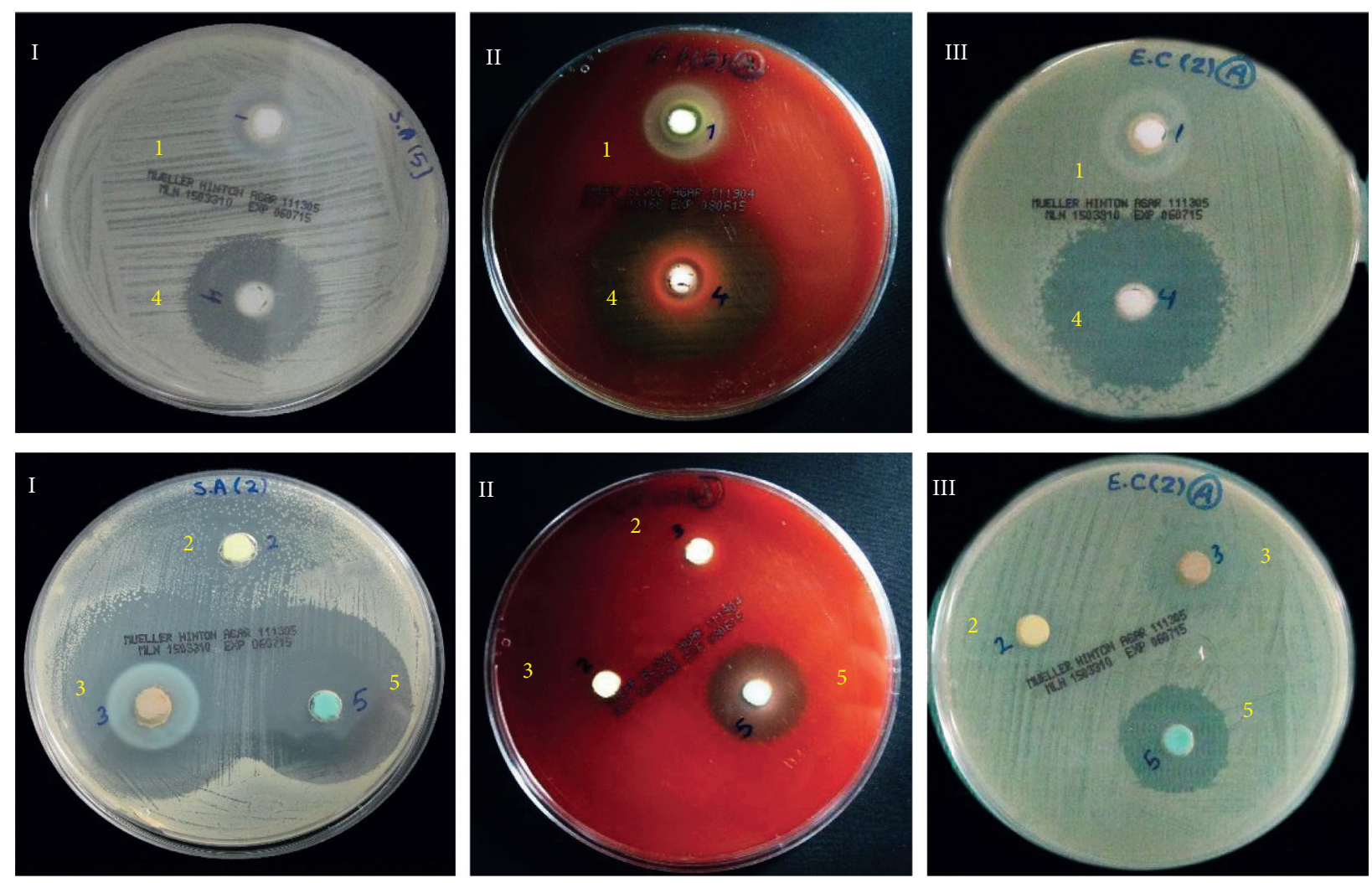

FiguRE 4: Zones of microbial growth inhibition around each experimental medicament for all tested microbial strains (Medicaments: 1: calcium hydroxide; 2: Diapex Plus; 3: Ledermix; 4: sodium hypochlorite gel; 5: CHX gel; Microbial strains: (I) S. aureus; (II) E. faecalis; (III) E. coli).

solution form [37, 38]. Up to our knowledge, there is no dental company producing the gel form of $\mathrm{NaOCl}$. However, the detergent companies produce this type of $\mathrm{NaOCl}$ as a bleaching material for clothes. Therefore, this study was conducted to evaluate and compare the antibacterial effect of sodium hypochlorite gel (Clorox Bleach Pen Gel) as a proposed intracanal medicament with that of the currently available root canal medicament such as chlorhexidine gel, calcium hydroxide paste, calcium hydroxide-iodoform combination (Diapex Plus), and antibiotic-corticosteroid paste (Ledermix).

In the present research, the agar diffusion test was chosen to assess the antimicrobial activity of the experimental medicaments because it is a simple technique by which the antimicrobial activity of endodontic materials can initially be assessed before carrying out more advanced tests [19]. It allows direct comparisons of the antimicrobial activity of endodontic materials by measuring the size of formed inhibition zones, indicating which material can eliminate possible microorganisms inside the root canal system. However, the size of the inhibition zones does not indicate the entire antimicrobial efficiency of the material [39]. The results may be influenced by many factors such as the chemical and physical properties of the tested material and culture medium [40]. In the current study, all attempts were made to decrease most of the variables such as type and thickness of agar medium, inoculums density, and incubation temperature. A Mueller-Hinton agar medium with a standardized thickness of $8 \mathrm{~mm}$ (Merck, Darmstadt, Germany) was used to ensure that all the agar plates had an equal thickness of agar [41]. The inoculum preparation was done similar to the method of Sipert et al. [42] and Asgary and Kamrani [43]. In order to ensure even distribution of the inoculums, the respective bacterial dilutions were swabbed uniformly onto the agar plates using lawn technique [44]. A well of $5 \mathrm{~mm}$ diameter and $4 \mathrm{~mm}$ depth was punched in each section using sterile glass Pasteur pipettes to ensure that the volume of each medicament was standardized [41, 44]. Finally, each zone of inhibition was measured with a millimeter ruler with an accuracy of $0.5 \mathrm{~mm}$ [43]. The results obtained from this in vitro test must be concluded with caution, because this test may not show the full clinical efficacy of the material being tested [45].

The null hypothesis of this study was rejected because the findings showed that there were significant differences between the tested medicaments regarding their antibacterial efficacy against all microbial strains.

The existing study revealed that $\mathrm{NaOCl}$ gel followed by $2 \%$ CHX gel had the uppermost antimicrobial activity against all tested microorganisms. This finding may be justified by the fact that $\mathrm{NaOCl}$ and $\mathrm{CHX}$ have a broad antibacterial spectrum and they are effective against both Gram-positive and Gram-negative bacteria [46]. However, the antibacterial effect of Ledermix against $S$. aureus was statistically similar to $\mathrm{CHX}$ gel and higher than $\mathrm{Ca}$ $(\mathrm{OH})_{2}$ paste. Meanwhile, $\mathrm{Ca}(\mathrm{OH})_{2}$ has shown better 
efficacy against $E$. faecalis than Ledermix. Regarding Diapex Plus, it did not show any antibacterial activity against all tested microbial strains. Among the tested microorganisms, E. faecalis was the most sensitive microorganisms to $\mathrm{NaOCl}$ gel and calcium hydroxide paste, while $S$. aureus was the most sensitive microorganism to CHX gel and Ledermix.

The current results complement the results of Jurczyk et al., who found that minimal inhibitory concentrations of $\mathrm{NaOCl}$ gel were equal to $\mathrm{CHX}$ solution against Gramnegative but higher against Gram-positive bacteria [47]. Shamsi et al. concluded that $5.25 \% \mathrm{NaOCl}$ gel exhibited the same effect as $5.25 \% \mathrm{NaOCl}$ solution against $E$. faecalis, and it might be recommended as an efficient intracanal irrigation agent [48]. However, Oncag et al. found that the 2\% CHX was significantly more powerful than $5.25 \% \mathrm{NaOCl}$ against E. faecalis [49].

On the contrary, the findings of the present study have shown that $\mathrm{NaOCl}$ gel was significantly more effective against all bacterial strains than CHX. Vianna et al. reported that $0.5 \%$ and $1 \% \mathrm{NaOCl}$ killed E. faecalis and S. aureus, within 20-30 minutes [50]. Other studies have shown that CHX gel was more effective against $E$. faecalis than the $\mathrm{Ca}$ $(\mathrm{OH})_{2}$ paste $[2,51,52]$ and can be used during the treatment of endodontic failure [53]. Gomes at al. showed that both gel and liquid formulation of 2\% CHX eliminated S. aureus in 15 $\mathrm{sec}$, while E. faecalis was killed by gel formulation in $1 \mathrm{~min}$ [54]. Despite the strong antibacterial action of $\mathrm{NaOCL}$, one of its major drawbacks in the root canal system is high surface tension, which limits its penetration into dentinal tubules and irregularities of the root canal system [55]. Moreover, its cytotoxicity is considered a clinical problem especially when it is extruded in the periapical area.

The bactericidal activity of sodium hypochlorite is due to the release of hypochlorous acid (HOCL) when added to water. The hypochlorous acid contains active chlorine, a potent oxidizing agent that allows irreversible oxidation of -SH groups of essential enzymes and thus disrupts the metabolic functions of the bacterial cell [56]. The antibacterial effect of chlorhexidine may be explained by the interaction of the positively charged chlorohexidine molecules and negatively charged phosphate groups on the bacterial cell wall. This interaction can alter the osmotic balance of the bacterial cell and allow the chlorohexidine molecule to penetrate the bacteria and produces direct toxic effect [16]. When chlorhexidine is used in high concentrations, there is a precipitation of cytoplasm, with consequent death of the microorganism [57]. It is better, in particular situations such as retreatment cases, to use antimicrobial agents that exhibit substantivity, that is, agents that can have a therapeutic effect for a prolonged period of time [26]. It has been demonstrated that CHX preparations, due to their cationic nature, exhibit the best substantivity among all endodontic agents [26, 58]. Depending on its concentration, CHX demonstrated substantivity from $48 \mathrm{~h}$ to 12 weeks in different studies $[59,60]$. Furthermore, it has been demonstrated that CHX, possibly due to its substantivity, may delay coronal leakage in endodontically treated teeth [30].
Some studies have revealed that $E$. faecalis was resistant to $\mathrm{Ca}(\mathrm{OH})_{2}$ medication [61]. Other studies indicated that calcium hydroxide was not very effective against endodontic biofilms [62] and bacteria penetrating dentinal tubules [63]. However, the findings of this study suggested that the calcium hydroxide paste demonstrated antimicrobial activity against all microbial strains but with a lower efficacy compared to $\mathrm{NaOCl}$ and $\mathrm{CHX}$ and this is in concurrence with the results of Ravishanker and Subba Rae [64]. Estrela et al. showed that $\mathrm{Ca}(\mathrm{OH})_{2}$ requires 60 days to produce antimicrobial activity against $E$. faecalis [65]. Zehnder et al. reported that $\mathrm{Ca}(\mathrm{OH})_{2}$ eliminated $E$. faecalis from radicular dentin after seven days [66]. Gomes et al. stated that $\mathrm{Ca}$ $(\mathrm{OH})_{2}$ has no uniform antimicrobial activity against all microorganisms present in the root canal and its interaction with antimicrobial substances becomes necessary [67].

The antimicrobial properties of $\mathrm{Ca}(\mathrm{OH})_{2}$ have been attributed to its high $\mathrm{pH}(11-12.5)$ and its dissociated hydroxyl ions, which destroy bacterial cells by protein denaturation and its detrimental effect on the cytoplasmic membrane and DNA [68]. Moreover, it serves as a physical barrier that can limit the ingress of microorganisms and impede the supply of nutrients to the remaining microorganisms, thereby preventing reinfection of the root canal and delaying recontamination [69].

The lower antimicrobial activity of $\mathrm{Ca}(\mathrm{OH})_{2}$ medication compared with $\mathrm{NaOCl}$ and $\mathrm{CHX}$ might be clarified by the proton pump property of the microorganisms $[61,70]$ and its delayed action [65]. In addition, the buffering agents in the culture medium can decrease the antibacterial effect of calcium hydroxide [71]. Additionally, some authors found that calcium hydroxide paste was not able to kill E. faecalis in the presence of dentine, hydroxyapatite, and bovine serum [72].

In certain situations, the alkaline environment in the radicular dentin created by calcium hydroxide may inhibit the resorptive activity of dentinoclasts, which needs an acid environment to achieve mineral dissolution. Hence, calcium hydroxide cannot be considered a universal intracanal medicament for all cases of infected root canal systems with apical periodontitis [73]. The current findings showed that Ledermix has an antimicrobial effect against E. coli and $S$. aureus but with little effect against E. faecalis, and that matches the results of Seow [74]. However, Athanassiadis et al. showed that the Ledermix and $\mathrm{Ca}(\mathrm{OH})_{2}$ were effective against E. faecalis [75].

Although iodoform is incorporated to improve the antibacterial properties of Diapex Plus [76], the results of the present research showed that this medicament has no antibacterial effect. This finding corresponds to the results of other authors [77]. Some authors found that the oil paste containing calcium hydroxide was mainly lacking both ion release and antibacterial properties [78]. Asmaa et al. [79] and Ravishanker and Subba Rae [64] concluded that Vitapex paste with $30 \% \mathrm{Ca}(\mathrm{OH})_{2}$ displayed the least inhibition of E. faecalis, while $1 \%$ CHX gel demonstrated better antibacterial efficacy.

Changes in the environment of microorganisms within the root canal system due to endodontic treatment 
may stimulate calcification of E. faecalis biofilms, as shown in a study by George et al. [80]. Those authors also reported that biofilms, formed under nutrient-rich conditions, had increased levels of calcium ions within the biofilm, while nutrient-poor conditions did not show this effect. This suggests a potential role of the mineralized matrix as a shelter for viable bacteria, which protects them from antimicrobial agents [80]. Further studies are therefore required to evaluate the antimicrobial effect of intracanal medicaments on the biofilms of different microorganisms.

A medicament that is effective in vitro against a single microbe may not necessarily be effective in vivo against the same microbe because the infected root canal consists of multiple microorganisms. Further clinical trials are required to validate the results of in vitro studies, addressing the effectiveness of medications against endodontic micro-organisms. Because of the excellent effectiveness of $\mathrm{NaOCl}$ gel against all tested microbial strains in the current study, it must be considered in the future clinical and laboratory researches aiming to release a strong intracanal medicament. Besides, dental manufacturers should take the present results of $\mathrm{NaOCl}$ gel in their considerations and improve its formula for use as an effective intracanal medicament. Until now, due to its excellent tissue biocompatibility and its intense antibacterial activity, CHX gel is considered the primary intracanal medicament that can be used effectively and safely until more laboratory and clinical studies on the $\mathrm{NaOCl}$ gel are conducted.

\section{Conclusions}

Within the limitations of the current in vitro study, the following conclusions can be drawn:

(1) Sodium hypochlorite gel and chlorhexidine gel demonstrated the strongest antibacterial activities among tested medicaments

(2) Calcium hydroxide paste exhibited antibacterial effect against all microbial strains particularly E. faecalis

(3) Ledermix showed a better antibacterial effect particularly against $S$. aureus and E. coli than calcium hydroxide but with lower antibacterial activity on E. faecalis

(4) Diapex Plus exhibited no antibacterial effect

(5) Sodium hypochlorite gel may be considered as an excellent intracanal medicament, and more laboratory and clinical trials are required to validate its antimicrobial effectiveness and safety

\section{Data Availability}

The data (measurements of inhibition zones) used to support the findings of this study will be available from the corresponding author elsayednada@yahoo.com for the researchers who meet the criteria for accessing this data. The data can be requested after the publication of this article. However, requests for the data (6/12 months) after the publication of this article will be considered by the corresponding author.

\section{Conflicts of Interest}

The authors declare that they have no conflicts of interest.

\section{Acknowledgments}

The authors would like to thank Ajman university and Thumbay Hospital, Ajman, UAE, for their support in the laboratory section of this manuscript.

\section{References}

[1] S. Kakehashi, H. R. Stanley, and R. J. Fitzgerald, "The effects of surgical exposures of dental pulps in germ-free and conventional laboratory rats," Oral Surgery, Oral Medicine, Oral Pathology, vol. 20, no. 3, pp. 340-349, 1965.

[2] Y. Lin, A. Mickel, and S. Chogle, "Effectiveness of selected materials against Enterococcus faecalis: Part 3. The antibacterial effect of calcium hydroxide and chlorhexidine on Enterococcus faecalis," Journal of Endodontics, vol. 29, no. 9, pp. 565-566, 2003.

[3] J. F. Siqueira and I. N. Rôças, Molecular Analysis of Endodontic Infections. Endodontic Microbiology. Fouad AF Ames, Wiley-Blackwell, Hoboken, NJ, USA, 2009.

[4] P. N. R. Nair, S. Henry, V. Cano, and J. Vera, "Microbial status of apical root canal system of human mandibular first molars with primary apical periodontitis after "one-visit" endodontic treatment," Oral Surgery, Oral Medicine, Oral Pathology, Oral Radiology, and Endodontology, vol. 99, no. 2, pp. 231-252, 2005.

[5] G. Sundqvist, "Ecology of the root canal flora," Journal of Endodontics, vol. 18, no. 9, pp. 427-430, 1992.

[6] G. Sundqvist, D. Figdor, S. Persson, and U. Sjögren, "Microbiologic analysis of teeth with failed endodontic treatment and the outcome of conservative re-treatment," Oral Surgery, Oral Medicine, Oral Pathology, Oral Radiology, and Endodontology, vol. 85, no. 1, pp. 86-93, 1998.

[7] L. Fabricius, G. Dahlén, S. E. Holm, and A. J. R. Möller, "Influence of combinations of oral bacteria on periapical tissues of monkeys," European Journal of Oral Sciences, vol. 90, no. 3, p. 200, 1982.

[8] C. M. Reader, M. Boniface, and S. Bujanda-Wagner, "Refractory endodontic lesion associated with Staphylococci aureus," Journal of Endodontics, vol. 20, no. 12, pp. 607-609, 1994.

[9] G. Pizzo, G. M. Giammanco, E. Cumbo, G. Nicolosi, and G. Gallina, "In vitro antibacterial activity of endodontic sealers," Journal of Dentistry, vol. 34, no. 1, pp. 35-40, 2006.

[10] T. G. Dwyer and M. Torabinejad, "Radiographic and histologic evaluation of the effect of endotoxin on the periapical tissues of the cat," Journal of Endodontics, vol. 7, no. 1, pp. 31-35, 1981.

[11] M. E. Vianna, H. P. Horz, B. P. F. A. Gomes, and G. Conrads, "In vivo evaluation of microbial reduction after chemo-mechanical preparation of human root canals containing necrotic pulp tissue," International Endodontic Journal, vol. 39, no. 6, pp. 484-492, 2006. 
[12] Aravind, V. Gopikrishna, D. Kandaswamy, and R. Jeyavel, "Comparative evaluation of the antimicrobial efficacy of five endodontic root canal sealers againstEnterococcus faecalisandCandida albicans," Journal of Conservative Dentistry, vol. 9, no. 1, pp. 2-12, 2006.

[13] L. B. Peters, A.-J. van Winkelhoff, J. F. Buijs, and P. R. Wesselink, "Effects of instrumentation, irrigation and dressing with calcium hydroxide on infection in pulpless teeth with periapical bone lesions," International Endodontic Journal, vol. 35, no. 1, pp. 13-21, 2002.

[14] R. M. Love, "Enterococcus faecalis-a mechanism for its role in endodontic failure," International Endodontic Journal, vol. 34, no. 5, pp. 399-405, 2001.

[15] P. V. Abbott, "Medicaments: aids to success in endodontics. Part 1. A review of the literature," Australian Dental Journal, vol. 35, no. 5, pp. 438-448, 1990.

[16] S. Lindskog, A. M. Pierce, and L. Blomlöf, "Chlorhexidine as a root canal medicament for treating inflammatory lesions in the periodontal space," Endodontics \& dental traumatology, vol. 14, pp. 186-190, 1998.

[17] https://www.thecloroxcompany.com/wpcontent/uploads/ cloroxbleachpengelforwhitesjw2014-09-21.pdf.

[18] M. Leonardo, L. Dasilva, M. Filho, K. Bonifácio, and I. Ito, "In vitro evaluation of antimicrobial activity of sealers and pastes used in endodontics," Journal of Endodontics, vol. 26, no. 7, pp. 391-394, 2000.

[19] J. H. Jorgensen and M. J. Ferraro, "Antimicrobial susceptibility testing: a review of general principles and contemporary practices," Clinical Infectious Diseases, vol. 49, no. 11, p. 1749 , 2009.

[20] A. Tonea, M. Badea, L. Oana, S. Sava, and D. Vodnar, "Antibacterial and antifungal activity of endodontic intracanal medications," Medicine and Pharmacy Reports, vol. 90, no. 3, pp. 344-347, 2017.

[21] G. S. P. Cheung and M. W. M. Ho, "Microbial flora of root canal-treated teeth associated with asymptomatic periapical radiolucent lesions," Oral Microbiology and Immunology, vol. 16, no. 6, pp. 332-337, 2001.

[22] M. R. Leonardo, F. F. Silveira, L. A. Silva, M. Tanomaru Filho, and L. S. Utrilla, "Calcium hydroxide root canal dressing. Histopathological evaluation of periapical repair at different time periods," Brazilian Dental Journal, vol. 13, no. 1, pp. 17-22, 2002.

[23] C. Estrela, F. C. Pimenta, I. Y. Ito, and L. L. Bammann, "Antimicrobial evaluation of calcium hydroxide in infected dentinal tubules," Journal of Endodontics, vol. 25, no. 6, pp. 416-418, 1999.

[24] I. Heling and M. Pecht, "Efficacy of Ledermix paste in eliminating Staphylococcus aureus from infected dentinal tubules in vitro," Dental Traumatology, vol. 7, no. 6, pp. 251-254, 1991.

[25] B. P. Gomes, M. E. Vianna, N. T. Sena, A. A. Zaia, C. C. Ferraz, and F. J de Souza Filho, "In vitro evaluation of the antimicrobial activity of calcium hydroxide combined with chlorohexidine gel used as intrcanal medication," Oral Surgery, Oral Medicine, Oral Pathology, Oral Radiology, and Endodontology, vol. 102, pp. 544-550, 2006.

[26] A. a. Khademi, Z. Mohammadi, and A. Havaee, "Evaluation of the antibacterial substantivity of several intra-canal agents," Australian Endodontic Journal, vol. 32, no. 3, pp. 112-115, 2006.

[27] J. Marley, D. Ferguson, and G. Hartwell, "Effects of chlorhexidine gluconate as an endodontic irrigant on the apical seal: short-term results," Journal of Endodontics, vol. 27, no. 12 , pp. $775-778,2001$.

[28] D. Ferguson, J. Marley, and G. Hartwell, "The effect of chlorhexidine gluconate as an endodontic irrigant on the apical seal: long-term results," Journal of Endodontics, vol. 29, no. 2, pp. 91-94, 2003.

[29] Z. Mohammadi and P. M. H. Dummer, "Properties and applications of calcium hydroxide in endodontics and dental traumatology," International Endodontic Journal, vol. 44, no. 8, pp. 697-730, 2011.

[30] B. P. F. A. Gomes, E. Sato, C. C. R. Ferraz, F. B. Teixeira, A. A. Zaia, and F. J. Souza-Filho, "Evaluation of time required for recontamination of coronally sealed canals medicated with calcium hydroxide and chlorhexidine," International Endodontic Journal, vol. 36, no. 9, pp. 604-609, 2003.

[31] Y.-L. Ng, V. Mann, and K. Gulabivala, "A prospective study of the factors affecting outcomes of nonsurgical root canal treatment: part 1: periapical health," International Endodontic Journal, vol. 44, no. 7, pp. 583-609, 2011.

[32] F. C. S. Chu, W. K. Leung, P. C. S. Tsang, T. W. Chow, and L. P. Samaranayake, "Identification of cultivable microorganisms from root canals with apical periodontitis following two-visit endodontic treatment with antibiotics/steroid or calcium hydroxide dressings," Journal of Endodontics, vol. 32, no. 1, pp. 17-23, 2006.

[33] H. M. A. Ahmed and P. V. Abbott, "Discolouration potential of endodontic procedures and materials: a review," International Endodontic Journal, vol. 45, no. 10, pp. 883-897, 2012.

[34] E. H. Ehrmann, H. H. Messer, and G. G. Adams, "The relationship of intracanal medicaments to postoperative pain in endodontics," International Endodontic Journal, vol. 36, no. 12, pp. 868-875, 2003.

[35] V. Zand, M. Lotfi, M. H. Soroush, A. A. Abdollahi, M. Sadeghi, and A. Mojadadi, "Antibacterial efficacy of different concentrations of sodium hypochlorite gel and solution on Enterococcus faecalis biofilm," Iranian Endodontic Journal, vol. 11, pp. 315-319, 2016.

[36] C. E. Radcliffe, L. Potouridou, R. Qureshi et al., "Antimicrobial activity of varying concentrations of sodium hypochlorite on the endodontic microorganisms Actinomyces israelii, A. naeslundii, Candida albicans and Enterococcus faecalis," International Endodontic Journal, vol. 37, no. 7, pp. 438-446, 2004.

[37] D. A. Al-Sudani and H. A. Al Omar, "Evaluation of sodium hypochlorite $(\mathrm{NaOCl})$ gel as an endodontic irrigant," Journal of Biomaterials and Tissue Engineering, vol. 1, no. 2, pp. 215-218, 2011.

[38] P. Mehra, C. Clancy, and J. Wu, "Formation of a facial hematoma during endodontic therapy," The Journal of the American Dental Association, vol. 131, no. 1, pp. 67-71, 2000.

[39] C. Estrela, C. Rodriguesdearaujoestrela, L. Luschkebammann, and J. Djalmapecora, "Two methods to evaluate the antimicrobial action of calcium hydroxide paste," Journal of Endodontics, vol. 27, no. 12, pp. 720-723, 2001.

[40] P. R. Murray, K. S. Rosenthal, G. S. Kobayashi, and M. A. Pfaller, Medical Microbiology, Mosby, St. Louis. USA, 4th edition, 2002.

[41] R. D. Morgental, F. V. Vier-Pelisser, S. D. Oliveira, F. C. Antunes, D. M. Cogo, and P. M. P. Kopper, "Antibacterial activity of two MTA-based root canal sealers," International Endodontic Journal, vol. 44, no. 12, pp. 1128-1133, 2011.

[42] C. R. Sipert, R. P. Hussne, C. K. Nishiyama, and S. A. Torres, "In vitro antimicrobial activity of fill canal, sealapex, mineral 
trioxide aggregate, portland cement and EndoRez," International Endodontic Journal, vol. 38, no. 8, pp. 539-543, 2005.

[43] S. Asgary and F. A. Kamrani, "Antibacterial effects of five different root canal sealing materials," Journal of Oral Science, vol. 50, no. 4, pp. 469-474, 2008.

[44] S. Saha, F. Samadi, J. Jaiswal, and U. Ghoshal, "Antimicrobial activity of different endodontic sealers: an in vitro evaluation," Journal of Indian Society of Pedodontics and Preventive Dentistry, vol. 28, no. 4, pp. 251-257, 2010.

[45] US Clinical and Laboratory Standards Institute, Performance Standards for Antimicrobial Susceptibility Testing. 17th Informational Supplement. CLSI Document M100-S17, US Clinical and Laboratory Standards Institute, Wayne, PA, USA, 2007.

[46] M. Haapasalo, U. Endal, H. Zandi, and J. Coil, "Eradication of endodontic infection by instrumentation and irrigation solutions," Endodontic Topics, vol. 10, pp. 71-102, 2005.

[47] K. Jurczyk, S. Nietzsche, C. Ender, A. Sculean, and S. Eick, "Invitro activity of sodium-hypochlorite gel on bacteria associated with periodontitis," Clinical Oral Investigations, vol. 20, no. 8, pp. 2165-2173, 2016.

[48] N. P. Shamsi, L. A. B. Yeganeh, B. V. Saberi, K. F. Parast, and A. T. Kashan, "Antibacterial effect of sodium hypochlorite gel and solution on Enterococcus faecalis," Journal of Dentomaxillofacial Radiology, Pathology, and Surgery, vol. 6, pp. 27-30, 2017.

[49] O. Onçă̆, M. Hoşgör, S. Hilmioğlu, O. Zekioğlu, C. Eronat, and D. Burhanoğlu, "Comparison of antibacterial and toxic effects of various root canal irrigants," International Endodontic Journal, vol. 36, no. 6, pp. 423-432, 2003.

[50] M. E. Vianna, B. P. F. A. Gomes, V. B. Berber, A. A. Zaia, C. C. R. Ferraz, and F. J. de Souza-Filho, "In vitro evaluation of the antimicrobial activity of chlorhexidine and sodium hypochlorite," Oral Surgery, Oral Medicine, Oral Pathology, Oral Radiology, and Endodontology, vol. 97, no. 1, pp. 79-84, 2004.

[51] O. Onçag, D. Cogulu, D. Gogulu, and A Uzel, "Efficacy of various intracanal medicaments against Enterococcus faecalis in primary teeth: an in vivo study," The Journal of Clinical Pediatric Dentistry, vol. 30, no. 3, pp. 233-237, 2006.

[52] R. J. Delgado, T. H. Gasparoto, C. R. Sipert et al., "Antimicrobial activity of calcium hydroxide and chlorhexidine on intratubular Candida albicans," International Journal of Oral Science, vol. 5, no. 1, pp. 32-36, 2013.

[53] V. Ballal, M. Kundabala, S. Acharya, and M. Ballal, "Antimicrobial action of calcium hydroxide, chlorhexidine and their combination on endodontic pathogens," Australian Dental Journal, vol. 52, no. 2, pp. 118-121, 2007.

[54] B. P. F. A. Gomes, C. C. R. Ferraz, V. B. Berber, F. B. Teixeira, and F. J. Souza-Filho, "In vitro antimicrobial activity of several concentrations of sodium hypochlorite and chlorhexidine gluconate in the elimination of Enterococcus faecalis," International Endodontic Journal, vol. 34, no. 6, pp. 424-428, 2001.

[55] F. Palazzi, M. Morra, Z. Mohammadi, S. Grandini, and L. Giardino, "Comparison of the surface tension of 5.25\% sodium hypochlorite solution with three new sodium hypochlorite-based endodontic irrigants," International Endodontic Journal, vol. 45, no. 2, pp. 129-135, 2012.

[56] G. Sirtes, T. Waltimo, M. Schaetzle, and M. Zehnder, "The effects of temperature on sodium hypochlorite short-term stability, pulp dissolution capacity, and antimicrobial efficacy," Journal of Endodontics, vol. 31, no. 9, pp. 669-671, 2005.

[57] B. P. Gomes, S. F. Souza, C. C. Ferraz et al., "Effectiveness of $2 \%$ chlorhexidine gel and calcium hydroxide against
Enterococcus faecalis in bovine root dentine in vitro," International Endodontic Journal, vol. 36, no. 4, pp. 267-275, 2003.

[58] S. Rosenthal, L. Spångberg, and K. Safavi, "Chlorhexidine substantivity in root canal dentin," Oral Surgery, Oral Medicine, Oral Pathology, Oral Radiology, and Endodontology, vol. 98, no. 4, pp. 488-492, 2004.

[59] R. R. White, G. L. Hays, and L. R. Janer, "Residual antimicrobial activity after canal irrigation with chlorhexidine," Journal of Endodontics, vol. 23, no. 4, pp. 229-231, 1997.

[60] M. R. Leonardo, M. Tanomaru Filho, L. A. Silva, P. Nelson Filho, K. C. Bonifácio, and I. Y. Ito, "In vivo antimicrobial activity of $2 \%$ chlorhexidine used as a root canal irrigation solution," Journal of Endodontics, vol. 25, pp. 167-171, 1999.

[61] M. Evans, J. K. Davies, G. Sundqvist, and D. Figdor, "Mechanisms involved in the resistance of Enterococcus faecalis to calcium hydroxide," International Endodontic Journal, vol. 35, no. 3, pp. 221-228, 2002.

[62] R. F. Zancan, R. R. Vivan, M. R. Milanda Lopes et al., "Antimicrobial activity and physicochemical properties of calcium hydroxide pastes used as intracanal medication," Journal of Endodontics, vol. 42, no. 12, pp. 1822-1828, 2016.

[63] J. F. Siqueira Jr and H. P. Lopes, "Mechanisms of antimicrobial activity of calcium hydroxide: a critical review," International Endodontic Journal, vol. 32, no. 5, pp. 361-369, 1999.

[64] P. Ravishanker and R. C. Subba, "Antimicrobial efficacy of four calcium hydroxide formulations and chlorhexidine gel using agar diffusion model," Internet Journal of Dental Science, vol. 8, pp. 1-5, 2009.

[65] C. Estrela, C. R. d. A. Estrela, and J. D. Pécora, "A study of the time necessary for calcium hydroxide to eliminate microorganisms in infected canals," Journal of Applied Oral Science, vol. 11, no. 2, pp. 133-137, 2003.

[66] M. Zehnder, M. Grawehr, G. Hasselgren, and T. Waltimo, "Tissue-dissolution capacity and dentin-disinfecting potential of calcium hydroxide mixed with irrigating solutions," Oral Surgery, Oral Medicine, Oral Pathology, Oral Radiology, and Endodontology, vol. 96, no. 5, pp. 608-613, 2003.

[67] B. P. F. d. A. Gomes, C. C. R. Ferraz, M. E. Vianna et al., "In vitro antimicrobial activity of calcium hydroxide pastes and their vehicles against selected microorganisms," Brazilian Dental Journal, vol. 13, no. 3, pp. 155-161, 2002.

[68] G. Tang, L. Samaranayake, and H.-K. Yip, "Molecular evaluation of residual endodontic microorganisms after instrumentation, irrigation and medication with either calcium hydroxide or Septomixine," Oral Diseases, vol. 10, no. 6, pp. 389-397, 2004.

[69] F. J. d. Souza-Filho, A. d. J. Soares, M. E. Vianna, A. A. Zaia, C. C. R. Ferraz, and B. P. F. d. A. Gomes, "Antimicrobial effect and $\mathrm{pH}$ of chlorhexidine gel and calcium hydroxide alone and associated with other materials," Brazilian Dental Journal, vol. 19, no. 1, pp. 28-33, 2008.

[70] T. M. T. Waltimo, D. Orstavik, E. K. Sirén, and M. P. P. Haapasalo, "In vitro susceptibility of Candida albicans to four disinfectants and their combinations," International Endodontic Journal, vol. 32, no. 6, pp. 421-429, 1999.

[71] S. Jhamb, V. Nikhil, and V. Singh, "An in vitro study of antibacterial effect of calcium hydroxide and chlorhexidine on Enterococcus faecalis," Indian Journal of Dental Research, vol. 21, no. 4, pp. 512-514, 2010.

[72] I. Porteiner, H. Haapasalo, A. Rye, T. Waltimo, D. Ørstavik, and M. Haapasalo, "Inactivation of root canal medicaments by dentine, hydroxyapatite and bovine serum albumin," International Endodontic Journal, vol. 34, pp. 184-188, 2001. 
[73] M. Torabinejad, B. Ung, and J. D. Kettering, "In vitro bacterial penetration of coronally unsealed endodontically treated teeth," Journal of Endodontics, vol. 16, no. 12, pp. 566-569, 1990.

[74] W. K. Seow, "The effects of dyadic combinations of endodontic medicaments on microbial growth inhibition," $P e$ diatric Dentistry, vol. 12, pp. 292-297, 1990.

[75] B. Athanassiadis, P. Abbott, N. George, and L. Walsh, "Anin vitrostudy of the antimicrobial activity of some endodontic medicaments and their bases using an agar well diffusion assay," Australian Dental Journal, vol. 54, no. 2, pp. 141-146, 2009.

[76] S. Gautam, B. Rajkumar, S. P. Landge, S. Dubey, P. Nehete, and L. C. Boruah, "Antimicrobial efficacy of metapex (calcium hydroxide with iodoform formulation) at different concentrations against selected microorganisms--an in vitro study," Nepal Medical College Journal :NMCJ, vol. 13, no. 4, pp. 297-300, 2011.

[77] C. Estrela, C. R. d. A. Estrela, A. C. B. Hollanda, D. d. A. Decurcio, and J. D. Pécora, "Influence of iodoform on antimicrobial potential of calcium hydroxide," Journal of Applied Oral Science, vol. 14, no. 1, pp. 33-37, 2006.

[78] P. Fulzele, S. Baliga, N. Thosar, and D. Pradhan, "Evaluation of calcium ion, hydroxyl ion release and $\mathrm{pH}$ levels in various calcium hydroxide based intracanal medicaments: an in vitro study," Contemporary Clinical Dentistry, vol. 2, no. 4, pp. 291-295, 2011.

[79] M. M. Asmaa, A. Alaa, El-Agamy, and I. K. Afifi, "Antimicrobial effect of different root canal medicaments on Enterococcus faecalis: in vitro comparative study," International Journal of Dentistry and Oral Science, vol. 1, pp. 15-20, 2014.

[80] S. George, A. Kishen, and P. Song, "The role of environmental changes on Monospecies biofilm formation on root canal wall by Enterococcus faecalis," Journal of Endodontics, vol. 31, no. 12, pp. 867-872, 2005. 